\title{
Tissue-Specific Regulation of Drosophila NF-kB Pathway Activation by Peptidoglycan Recognition Protein SC
}

\author{
Denis Costechareyre $^{a}$ Florence Capo $^{a} \quad$ Alexandre Fabre $^{b} d \quad$ Delphine Chaduli $^{a}$ \\ Christine Kellenberger $^{c} \quad$ Alain Roussel $^{c}$ Bernard Charroux $^{a} \quad$ Julien Royet $^{a}$ \\ anstitut de Biologie du Développement de Marseille, UMR 7288, CNRS, b Génétique Médicale et Génomique \\ Fonctionnelle, Faculté de Médecine, INSERM, UMR_S 910, and ' Laboratoire Architecture et Fonction des \\ Macromolécules, UMR 7257, Centre National de la Recherche Scientifique, Aix Marseille Université, and \\ d Service de Pédiatrie Multidisciplinaire, Hôpital d'Enfants de la Timone, Assistance Publique, Hôpitaux de Marseille, \\ Marseille, France
}

\section{Key Words}

Amidase $\cdot$ Drosophila $\cdot$ Immune deficiency $\cdot$ Innate immunity · Nuclear factor-KB · Peptidoglycan .

Peptidoglycan recognition proteins $\cdot$ Toll signaling

\begin{abstract}
In Drosophila, peptidoglycan (PGN) is detected by PGN recognition proteins (PGRPs) that act as pattern recognition receptors. Some PGRPs such as PGRP-LB or PGRP-SCs are able to cleave PGN, therefore reducing the amount of immune elicitors and dampening immune deficiency (IMD) pathway activation. The precise role of PGRP-SC is less well defined because the PGRP-SC genes (PGRP-SC1a, PGRP$S C 1 b$ and $P G R P-S C 2$ ) lie very close on the chromosome and have been studied using a deletion encompassing the three genes. By generating PGRP-SC-specific mutants, we reevaluated the roles of PGRP-LB, PGRP-SC1 and PGRP-SC2, respectively, during immune responses. We showed that these genes are expressed in different gut domains and that they follow distinct transcriptional regulation. Loss-of-function mutant analysis indicates that PGRP-LB is playing a ma-
\end{abstract}

\section{KARGER}

E-Mail karger@karger.com

www.karger.com/jin jor role in IMD pathway activation and bacterial load regulation in the gut, although PGRP-SCs are expressed at high levels in this organ. We also demonstrated that PGRP-SC2 is the main negative regulator of IMD pathway activation in the fat body. Accordingly, we showed that mutants for either PGRP-LB or PGRP-SC2 displayed a distinct susceptibility to bacteria depending on the infection route. Lastly, we demonstrated that PGRP-SC1 and PGRP-SC2 are required in vivo for full Toll pathway activation by Gram-positive bacteria.

(c) 2015 S. Karger AG, Basel

\section{Introduction}

Peptidoglycan (PGN) and PGN recognition proteins (PGRPs) are the main microbe-associated molecular patterns and pattern recognition receptors that regulate the antibacterial response in Drosophila, respectively [14]. Some PGRP family members such as PGRP-LC, PGRP-SA, PGRP-SD or PGRP-LE have the ability to bind PGN and are therefore essential sentinels upstream 
of the two NF- $\kappa \mathrm{B}$-dependent Drosophila signaling cascades, called Toll and immune deficiency (IMD) [4-15]. Recognition of lysine (Lys)-type PGN by PGRP-SA is sufficient to trigger the Toll/Dorsal/Dif signaling, whereas detection of diaminopimelic (DAP)-type PGN (either membrane-associated via PGRP-LC or intracellularly via PGRP-LE) promotes IMD/Relish signaling activation $[16,17]$.

Biochemical experiments have demonstrated that other PGRP family members, such as PGRP-LB, PGRP-SB and PGRP-SC, are not only able to bind PGN but also display an amidasic activity that allows them to cleave PGN into smaller nonimmunogenic muropeptides [1820]. In the case of PGRP-LB, in vivo experiments have clearly shown that by degrading PGN, PGRP-LB provides a negative feedback regulation that allows a tight adjustment of the immune activation to the intensity of the infection $[18,21]$. In the absence of PGRP-LB, flies overrespond to bacteria and eventually die for unknown reasons. Although the ability of PGRP-SC proteins to cleave PGN is clearly documented, published results on the in vivo role of PGRP-SC in immune system activation are difficult to reconcile into a coherent model.

The PGRP-SC1 protein is coded by two genes, PGRPSC1a and PGRP-SC1b, which both produce the same polypeptide. This amidase was initially identified as a scavenger receptor that, by cleaving Staphylococcus aureus Lys-type PGN, reduces its immune-stimulatory activity on the IMD pathway in cultured cells [22]. Later on, using an RNAi-mediated approach, it was shown that simultaneous inactivation of PGRP-SC1a/PGRP-SC1b and PGRP-SC2 in the gut induces ectopic expression of immune-inducible genes in the fat body following Escherichia coli ingestion [23]. This role of PGRP-SCs as negative regulators of IMD pathway activation was later confirmed using a deletion removing PGRP-SC1a/ PGRP-SC1b and PGRP-SC2 [21]. This study also revealed that PGRP-SC-dependent negative regulation takes place in the fat body during the systemic response and not in the gut itself. PGRP-SC1 was independently identified through an EMS genetic screen as a protein required for Toll pathway activation and for phagocytosis [24]. Surprisingly, while the PGN-cleaving activity is required to mediate $S$. aureus phagocytosis, it is dispensable for Toll activation. Finally, a recent report proposed that by reducing IMD/Relish signaling in the gut, PGRP-SC2 is preventing commensal dysbiosis, stem cell hyperproliferation and epithelial dysplasia, and, in turn, prevents gut aging [25]. The different conclusions drawn from these studies could be explained, at least partly, either by the different techniques used to inactivate the genes (RNAi, $\mathrm{KO}$ or EMS, for example) or by the fact that while some studies analyzed the effect of removing one PGRP-SC (PGRP-SC1 or PGRP-SC2), others described the phenotype of Drosophila mutant affecting both PGRP-SC1 and PGRP-SC2.

To clarify the respective role of PGRP-SCs and PGRP$\mathrm{LB}$ in immune response modulation, we generated specific KO for each of the PGRP-SC genes and analyzed their immune phenotypes. Our results failed to identify any clear IMD-dependent function for PGRP-SC1, although its transcriptional induction is the highest of the entire genome after gut bacterial colonization. We demonstrated that although PGRP-SC2 and PGRP-LB are both strong negative regulators of IMD, they act in different tissues. Whereas PGRP-LB is needed in the gut to cleave PGN and prevent both local gut activation and PGN dissemination into the hemolymph, PGRP-SC2 is mainly required in the fat body to control systemic immune response. Rescue experiments also show that PGRP-SC2 and PGRP-LB are not functionally equivalent. Finally, mutant phenotype analysis indicated that both PGRP-SC1 and PGRP-SC2 are positive regulators of the Toll signaling cascade.

\section{Materials and Methods}

\section{Bacterial Strains}

The following microorganisms were used: Lactobacillus plantarum $^{\text {WJL }}$, Erwinia carotovora carotovora 152141 (Ecc), Micrococcus luteus and Enterococcus faecalis. All strains were cultured in LuriaBertani medium, except L. plantarum (MRS medium). L. plantarum and E. faecalis were cultured at $37^{\circ} \mathrm{C} ; \mathrm{Ecc}$ and M. luteus at $30^{\circ} \mathrm{C}$.

\section{D. melanogaster Strains and Maintenance}

The following strains were used in this work: Oregon-R (WT), SC1a-Gal4 (this work), SC1b-mCherry (this work), UAS-nlsGFP BL No. 4775, imd shadok [15], PGRP-LE ${ }^{112}$ [7], PGRP-LC ${ }^{\Delta E 12}$ [15], SC2-Gal4 (this work), PGRP-SC1a/1 $b^{-/-}$(this work) and PGRP$S C 2^{-/-} \mathrm{KOs}$ (this work).

Flies were grown at $25^{\circ} \mathrm{C}$ on a yeast/cornmeal medium. For 1 liter of food, 8.2 g of agar (VWR, cat. No. 20768.361), $80 \mathrm{~g}$ of cornmeal flour (Westhove, Farigel Maize H1) and $80 \mathrm{~g}$ of yeast extract (VWR, cat. No. 24979.413) were cooked for $10 \mathrm{~min}$ in boiling water; $5.2 \mathrm{~g}$ of methylparaben sodium salt (Merck, cat. No. 106756) and $4 \mathrm{ml}$ of $99 \%$ propionic acid (Carlo Erba, cat. No. 409553) were added when the food had cooled down. For antibiotic treatment, standard medium was supplemented with ampicillin, kanamycin, tetracycline and erythromycin at final concentrations of $50 \mu \mathrm{g} / \mathrm{ml}$. For all tests, the adult flies used were exclusively 6-day-old females.

\section{Mutant Generation}

PGRP-SC1a/1 $b^{-/-}$and PGRP-SC2 ${ }^{-/-}$KO lines were generated by homologous recombination. Gene clusters were replaced by a
68

J Innate Immun 2016;8:67-80 DOI: $10.1159 / 000437368$
Costechareyre/Capo/Fabre/Chaduli/ Kellenberger/Roussel/Charroux/Royet 
mini-white gene. DNA flanking the $5^{\prime}$ and $3^{\prime}$ ends used were 3,041 and 2,982 bp for the PGRP-SC1a/1b locus, and 3,002 and 2,980 bp for the PGRP-SC2 locus, respectively. Sequences were cloned into the pW25 vector [26].

\section{Monoassociation of Germ-Free Flies with L. plantarum}

Germ-free embryos laid on standard culture medium by germfree females were covered with $150 \mathrm{ml} \mathrm{L}$. plantarum suspension with an optical density (OD) of 2. Emerging larvae were allowed to develop on the contaminated media. Third-instar larvae (96 h after egg laying) were then dissected.

\section{Natural Infection of Adults by Ecc-15}

Overnight bacterial cultures were centrifuged at 2,500 $g$ for 8 $\mathrm{min}$ at room temperature and resuspended in fresh Luria-Bertani medium. Cells were serially diluted in PBS and their concentration was determined by OD measurement at $600 \mathrm{~nm}$. For oral infection, flies were first incubated for $2 \mathrm{~h}$ at $29^{\circ} \mathrm{C}$ in empty vials and then placed in a fly vial with food. The food solution was obtained by mixing a pellet of an overnight culture of bacterial Ecc-15 (OD = $200)$ with a solution of $5 \%$ sucrose $(50 / 50)$ and added to a filter disk that completely covered the agar surface of the fly vial. Septic injuries were performed by pricking adult females with a thin needle contaminated with Ecc-15.

\section{Bacterial Loads}

The bacterial load of surface-sterilized individuals was quantified by plating serial dilutions of lysates obtained from 10 individuals on nutrient agar plates (MRS for L. plantarum). Homogenization of individuals was performed using the Precellys 24-tissue homogenizer (Bertin Technologies, France) and 0.75-/1-mm glass beads in $800 \mathrm{ml}$ of the appropriate bacterial culture medium. (Bacterial loads were analyzed $72 \mathrm{~h}$ after monoassociation in larval flies.)

Survival Tests with Bacterial Infection

Orally induced bacterial infections were performed with Pseudomonas entomophila. An overnight culture was centrifuged at $2,500 \mathrm{~g}$ for $5 \mathrm{~min}$ at room temperature and resuspended at OD = 1 in fresh Luria-Bertani medium with $2.5 \%$ of sucrose. This bacterial solution was deposited on a filter disk that completely covered the agar surface of the fly vial and flies were added.

Systemic infections (septic injury) were performed with $P$. entomophila or E. faecalis by pricking adult females in the thorax with a thin needle previously dipped into a concentrated pellet of the bacterial culture. Infected flies were subsequently maintained at $29^{\circ} \mathrm{C}$. At least two tubes of 20 flies were used for each survival assay and three replicates of this experiment were done.

Mutants and control populations are compared using the logrank and the Wilcoxon test ( $\chi^{2}$ distribution and $\mathrm{p}$ values). All analyses were performed using GraphPad Prism 6 statistical software.

\section{Imaging}

Larval or adult tissues were dissected in PBS and fixed for 20 min in $4 \%$ paraformaldehyde on ice. After several rinses in PBT $(\mathrm{PBS}+0.1 \%$ Triton $\mathrm{X}-100)$, the tissues were mounted in Vectashield (Vector Laboratories) fluorescent mounting medium with DAPI. Images were captured with either a Stereo Discovery V12 microscope or an LSM 780 Zeiss confocal microscope.

Tissue-Specific Regulation of Immune

Responses by Amidases

\section{Quantitative Real-Time PCR}

RNAs from entire flies $(n=12)$, guts $(n=12)$, fat bodies $(n=$ 12) or cultured S2 cells $(300 \mu \mathrm{l})$ were extracted with the RNeasy mini kit (Qiagen, cat. No. 74106). Quantitative real-time PCR, TaqMan and SYBR Green analyses were performed as previously described [27]. Information on primers can be obtained upon request. The amount of mRNA detected was normalized to control rp49 mRNA values. Normalized data were used to quantify the relative levels of a given mRNA according to cycle threshold analysis $\left(\Delta \mathrm{C}_{\mathrm{T}}\right)$.

\section{Drosophila S2 Cells for PGRP-SC Expression}

cDNA fragments corresponding to the whole coding sequences of PGRP-SC1a and PGRP-SC2 were amplified using specific oligonucleotides. The PCR-amplified DNAs were digested by the appropriate restriction enzymes and then inserted at the specific sites into the vector pMT/BIP/V5-HisA (Life Technologies, cat. No. V4120-20). The resulting recombinant plasmids encode for V5-His-tagged proteins. The integrity of all constructs was assessed by DNA sequencing. The recombinant plasmids encoding PGRP-SC1 and PGRP-SC2 were cotransfected with the pAc5C-pac vector (an actin5C-driven expression vector for puromycin acetyltransferase) into Drosophila S2 cells according to the protocol from Life Technologies. Stable clones were obtained using puromycin selection. Cells were grown in suspension at $23^{\circ} \mathrm{C}$ at a cell density of $3-4 \times 10^{6}$ cells $/ \mathrm{ml}$ and kept under selection in Schneider's medium (Sigma) containing 0.5 $\mu \mathrm{g} / \mathrm{ml}$ puromycin (Life Technologies), $50 \mu \mathrm{g} / \mathrm{ml}$ streptomycin (Gibco), $50 \mu \mathrm{g} / \mathrm{ml}$ penicillin (Gibco) and $10 \%$ heat-inactivated fetal bovine serum (Gibco). For immune tests with bacteria, cells were diluted at a cell density of $10^{6}$ cells $/ \mathrm{ml}$ on day 1 . On day 2 , expression of the proteins was induced by addition of $0.05 \mathrm{~mm}$ $\mathrm{CuSO}_{4}$, and the contact with dead bacteria was done on day 3 for $24 \mathrm{~h}$.

\section{Results}

\section{PGRP-SC1 $a$ and PGRP-SC1b Are Expressed in \\ Overlapping Domains in the Larval Gut}

The PGRP-SC1 $a$ and PGRP-SC1b genes lie $3 \mathrm{~kb}$ apart on the second chromosome and code for two identical polypeptides [28]. To reveal their expression pattern, we generated reporter lines in which $1.5 \mathrm{~kb}$ of genomic DNA $5^{\prime}$ of each coding region was cloned upstream to either mCherry (for PGRP-SC1b)- or Gal4-coding sequences (for PGRP-SC1a). When raised in axenic condition, transgenic PGRP-SC1a-mCherry or PGRP-SC1b-Gal4, $U A S-n l s G F P$ larvae did not display fluorescent signals in the intestinal tract (data not shown). When larvae of the same genotypes were fed with the commensal strain, $L$. plantarum, both transgenes were highly and specifically expressed in the anterior part of the posterior midgut (Pmg) in almost completely overlapping domains (fig. 1a, b). Very similar expression patterns were observed for the 

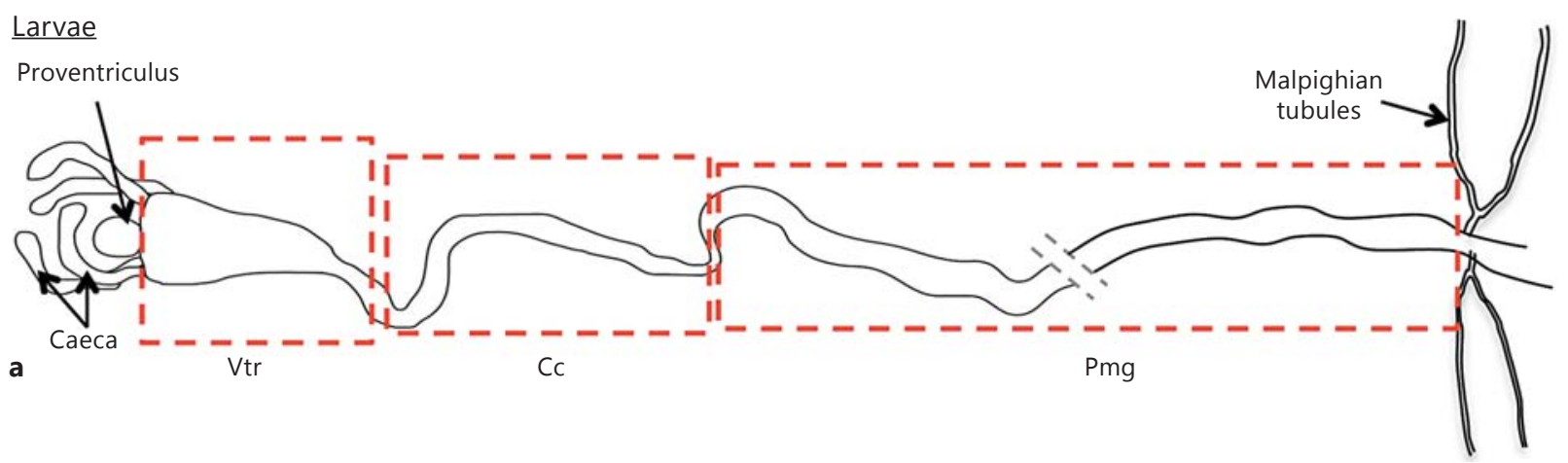

Larvae
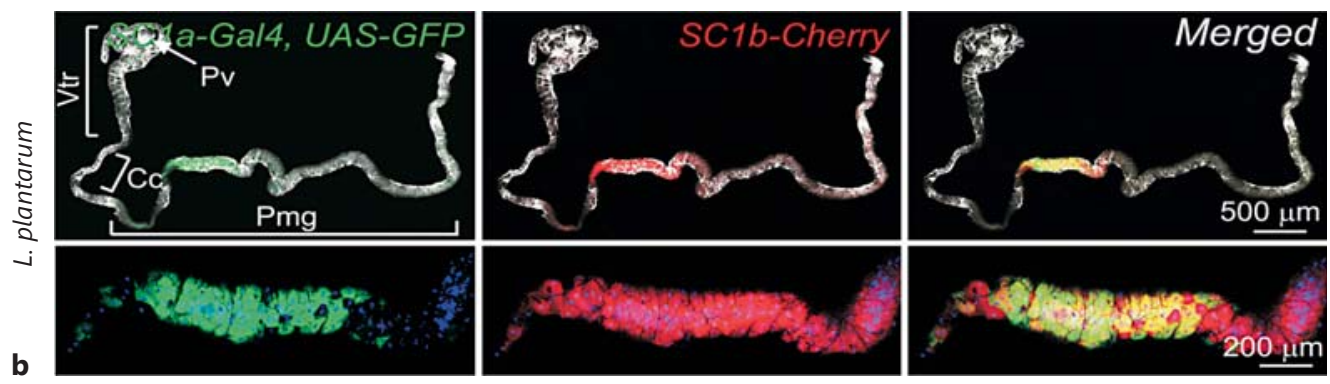

Larvae

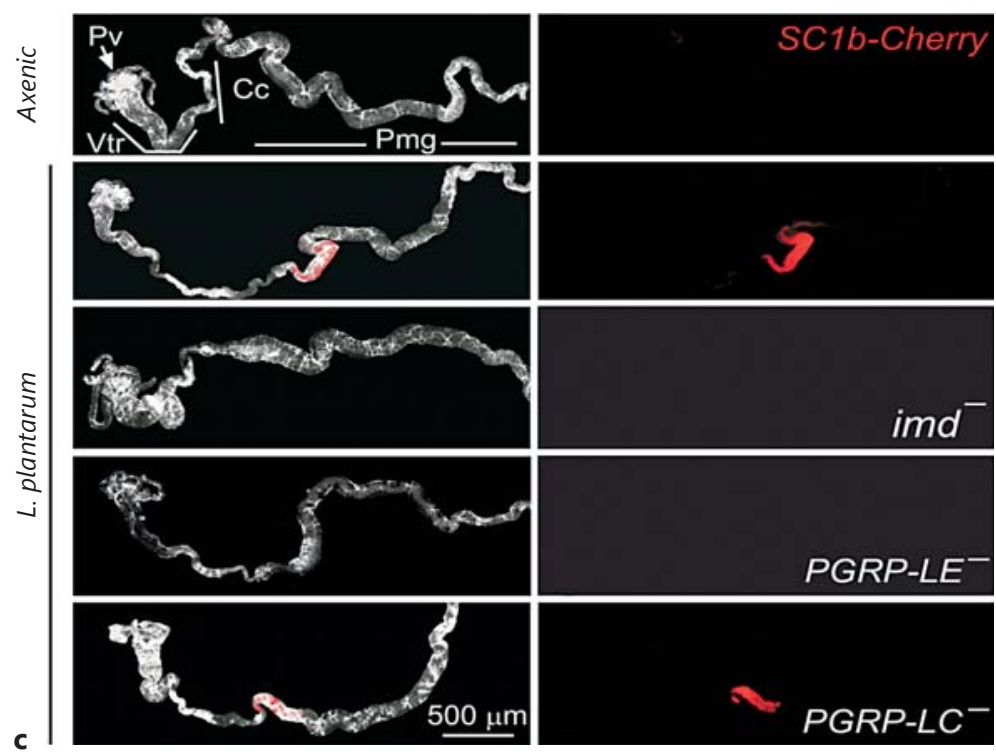

Adult
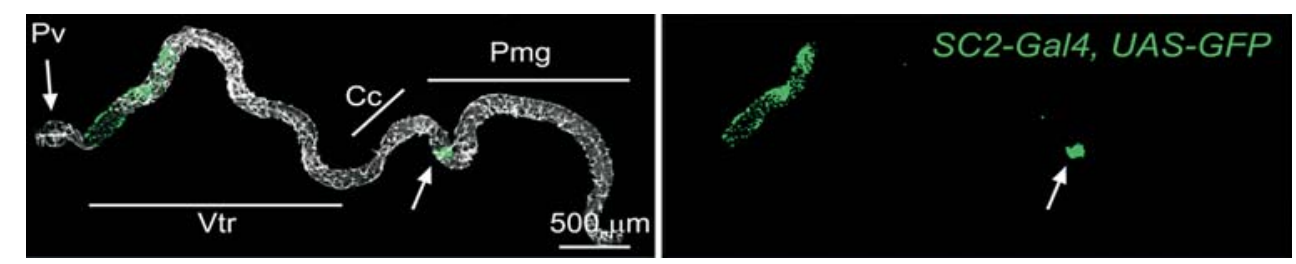

(For legend see next page.) 

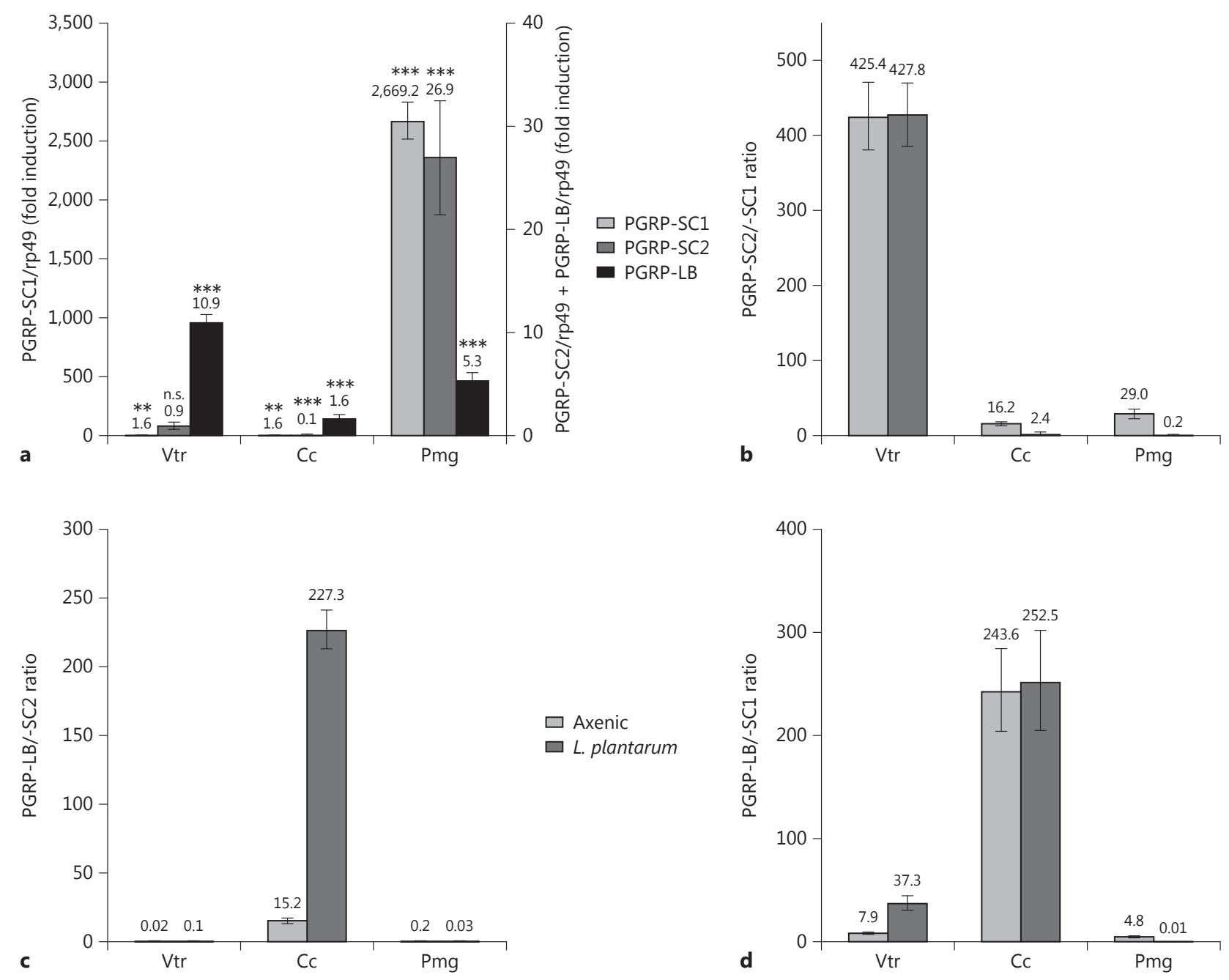

Fig. 2. PGRP mRNA levels in L. plantarum-colonized gut domains. a Induction levels of PGRP-SC1, PGRP-SC2 and PGRP-LB mRNAs in gut domains of $L$. plantarum-colonized larvae compared to axenic larvae (= control value). Means \pm SD of three independent experiments. Statistical significance of the results with
Student's t test analysis is included: $\mathrm{p}>0.05$ (n.s.), $* * \mathrm{p}<0.05$ and *** $\mathrm{p}<0.01$. b-d Relative ratios between PGRP-SC2 and PGRP$S C 1$ (b), between PGRP-LB and PGRP-SC2 (c) and between PGRP$L B$ and PGRP-SC1 (d) mRNAs in different gut domains of axenic and L. plantarum-colonized larvae.
Fig. 1. Expression pattern of $P G R P-S C 1 a, P G R P-S C 1 b$ and $P G R P$ $S C 2$ in the larval gut. Colors refer to the online version only. a Schematic representation of a dissected larval gut. b PGRP-SC1a and $P G R P-S C 1 b$ expression pattern in the larval gut of flies associated with L. plantarum. PGRP-SC1a-Gal4, UAS-nlsGFP (green) and PGRP-SC1b-mCherry (red) are coexpressed in the anterior part of the midgut. c IMD and PGRP-LE, but not PGRP-LC, are required for $P G R P-S C 1$ expression in the gut. Confocal pictures of axenic or L. plantarum-associated larval guts of the following genotypes: SC1b-Cherry (PGRP-SC1b-mCherry), imd ${ }^{-}$(PGRP-SC1bCherry, imd shadok $^{\text {s. } P G R P-L E^{-}(P G R P-S C 1 b-m C h e r r y ;}$ PGRP-LE $\left.{ }^{112}\right)$ and PGRP-LC $\left(\right.$ PGRP-SC1b-mCherry; PGRP-LC $\left.C^{E 12}\right)$.d PGRP-SC2 expression in adult guts of axenic flies. PGRP-SC2-Gal4, UAS-nlsGFP (green) is expressed in Vtr and in a narrow domain of the Pmg. Pv $=$ Proventriculus. 
PGRP-SC1 transgenes in adult infected guts (data not shown). This indicated that these two genes that code for the same protein and are expressed in very similar domains are transcriptionally regulated by two different promoter regions. To insure that the lines were faithfully reporting endogenous gene expression patterns, PGRPSCla-mCherry flies were crossed to IMD pathway mutants known to regulate its transcription. As expected from previous work [29], PGRP-SC1 gene activation upon $L$. plantarum gut colonization required a functional PGRP-LE receptor and an IMD adaptor but was PGRP-LC independent (fig. 1c).

PGRP-SC1, PGRP-SC2 and PGRP-LB Have Different Expression Patterns in the Gut

Previous work has shown that PGRP-SC1, PGRP-SC2 and $P G R P-L B$ are all expressed in the gut [21]. We then decided to quantify the relative expression levels of $P G R P$ $S C 1 a / P G R P-S C 1 b, P G R P-S C 2$ and PGRP-LB mRNAs in different domains of naive and $L$. plantarum-colonized midguts (fig. 2a). While PGRP-SC1a/PGRP-SC1b and $P G R P-S C 2$ transcription were not modified upon L. plantarum recolonization in ventriculus (Vtr) and copper cells $(\mathrm{Cc})$, it was strongly up-regulated in the Pmg of bacterium-colonized guts. PGRP-SC2 induction was, however, a hundred times lower than that of PGRP-SC1 (fig. 2a). This contrasted with $P G R P-L B$ mRNAs, whose levels were only moderately affected by the presence of bacteria with a 10 and 5 times increase in the Vtr and Pmg, respectively. We then compared the relative abundance of the three amidase mRNAs in the different gut domains contaminated or not with bacteria (fig. 2b-d). In axenic larvae, PGRP-SC2 mRNAs were more abundant than PGRP-SC1 mRNAs in all gut domains, but most dramatically in the anterior Vtr, in which the PGRP-SC2/ $P G R P-S C 1$ ratio can be increased up to 400 times (fig. 2b). These results indicated that the pattern and dynamics of PGRP-SC1a/PGRP-SC1b and PGRP-SC2 expression are very different. Whereas PGRP-SC1a/PGRP-SC1b are transcribed at very low levels in axenic guts and are strongly up-regulated in the Pmg after bacterial colonization, PGRP-SC2 expression is constitutive in anterior domains and moderately modified by bacteria in the Pmg. A similar pattern of expression was observed at the adult stage using a PGRP-SC2-Gal4, UAS-nlsGFP reporter line (fig. 1d). In contrast to PGRP-SC1a-mCherry lines in which the signal was only detected in the presence of bacteria, the GFP signal in the PGRP-SC2-Gal4, UAS-nlsGFP line was already observed in axenic conditions (fig. 1d) and not strongly modified by bacteria (not shown). These data indicated that although the three amidases are expressed in the gut, their specific domain of expression, their relative ratio and their induction upon bacterial infection are unique to a given amidase. In other words, each gut domain contains a specific cocktail of amidases that is either constitutively expressed or induced by the presence of microorganisms.

Generating Single PGRP-SC1 and PGRP-SC2 Mutants The fact that each amidase displays a unique transcription pattern suggested that they might play a distinct role in the gut immune response. In order to further dissect their respective contribution to immune responses in the gut but also in other immune tissues, we took advantage of a previously generated $P G R P-L B^{-/-}$mutant and have generated $P G R P-S C 1^{-/-}$and $P G R P-S C 2^{-/-}$-specific $\mathrm{KOs}$ through homologous recombination (fig. 3a) [21]. Since the CG14743 gene is inserted in between PGRP-SC1 $a$ and $P G R P-S C 1 b$, the $P G R P-S C 1^{-/-}$construct also removed it (fig. 3a). Our results demonstrated that this gene is not expressed in the gut (data not shown), confirming previously published data showing that its inactivation does not affect immune responses [21]. As expected, quantitative real-time PCR on dissected adult gut domains showed that PGRP-SC1 and PGRP-SC2 mRNAs were absent in their respective $\mathrm{KO}$ mutant, thereby validating the tools generated (fig. 3b, c). However, the proximity of the two genes on the chromosome led us to test whether genome editing performed at one locus could impact gene expression at the neighboring locus. In axenic guts, basal PGRP$S C 1$ transcription was slightly affected by the absence of PGRP-SC2 (fig. 3b). The effects were stronger with the reciprocal combination with a $70-80 \%$ decrease in the $P G R P-S C 2$ mRNA level in PGRP-SC1 ${ }^{-/}$. However, since $P G R P-S C 2$ is a gene whose basal expression is high in the gut, the PGRP-SC1 ${ }^{-/}$mutant cannot be considered as a double mutant. Moreover, in other tissues such as the fat body, PGRP-SC1 inactivation had no influence on PGRP$S C 2$ expression and vice versa (online suppl. fig. S1; see www.karger.com/doi/10.1159/437368 for all online suppl. material). Finally, $P G R P-L B$ inactivation was associated with a slight increase in PGRP-SC1 and PGRP-SC2 mRNA levels (fig. 3b, c; online suppl. fig. S1). In contrast, $P G R P-S C$ elimination had only minor effects on PGRP$L B$ expression (fig. 3d).

\section{Amidase Inactivation Alters Gut IMD Pathway Activation by L. plantarum}

Taking advantage of the newly generated mutants, we tested the implication of each amidase for gut immune
Costechareyre/Capo/Fabre/Chaduli/ Kellenberger/Roussel/Charroux/Royet 


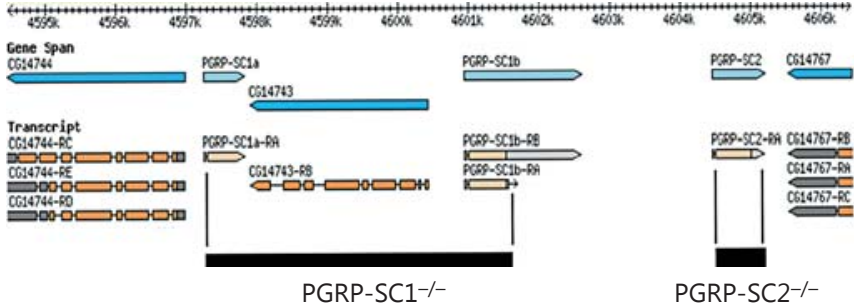

a

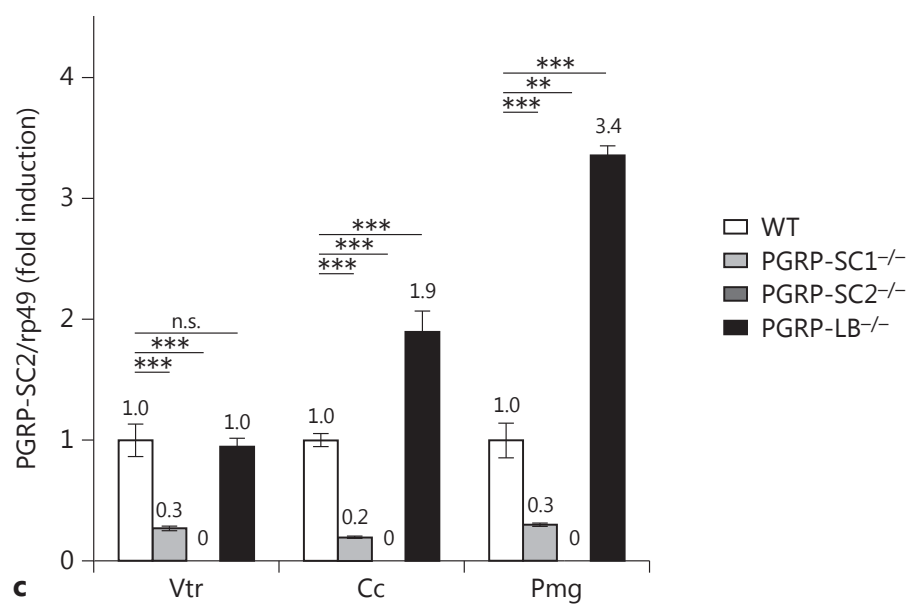

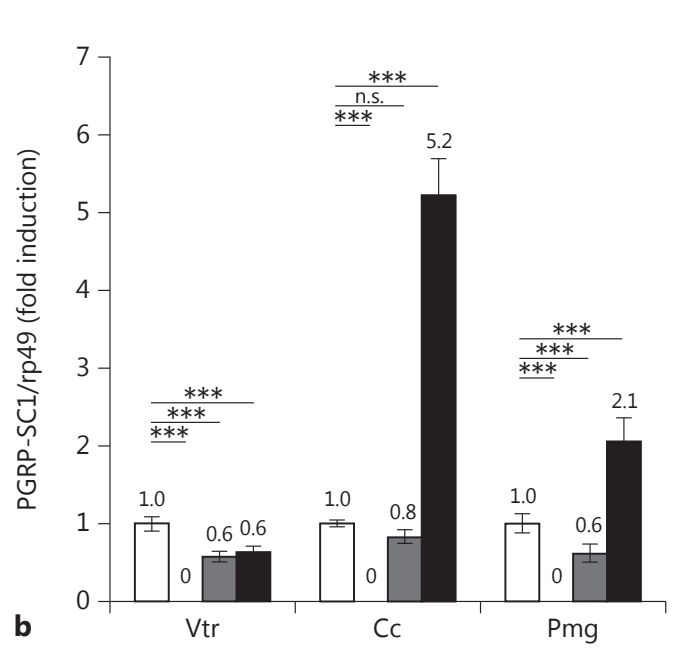

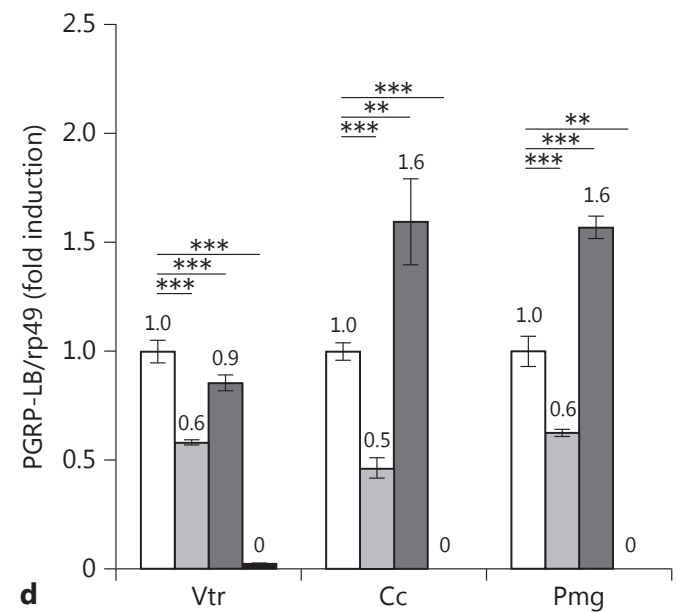

Fig. 3. Generation and characterization of PGRP-SC1- and PGRP$S C 2$-specific mutants. a Schematic representation of $P G R P-S C 1 a / b$ and PGRP-SC2 mutants. The gene map was adapted from FlyBase. The deleted segment replaced by the mini-white gene (black box) is indicated. For the PGRP-SC1a/b locus, the deletion starts at position 2R: 8,709,733 and ends at position 2R: 8,715,094 and also includes the CG14743 gene; for the PGRP-SC2 locus, the deletion

responses following colonization with $L$. plantarum, bacteria previously shown to be tolerated by the gut immune system [30]. Using attacin D (AttD) as a molecular readout of the IMD pathway, we showed that amidase inactivation had domain-specific effects (fig. 4a). Although inactivation of any of the three amidases provoked an up-regulation of $A t t D$ expression in the Vtr, the strongest effects were seen in PGRP-LB $B^{-/-}$. Since PGRP-SC1 mRNAs were almost absent in the Vtr and

Tissue-Specific Regulation of Immune Responses by Amidases starts at position 2R: 8,716,950 and ends at position 2R: 8,717,695. b-d Relative gene expression of PGRP-SC1 (b), PGRP-SC2 (c) and $P G R P-L B(\mathbf{d})$ in different gut domains of axenic larvae of the three specific mutants compared to controls (WT). Means \pm SD of three independent experiments. $\mathrm{p}>0.05$ (n.s.), ${ }^{* *} \mathrm{p}<0.05$ and ${ }^{* * *} \mathrm{p}<$ 0.01 (Student's t test).

since $P G R P-S C 1^{-/-}$strongly reduced the PGRP-SC2 mRNA level, the effects seen in PGRP-SC1 ${ }^{-/-}$could be secondary to a reduction in PGRP-SC2 mRNAs. Conversely, $A t t D$ expression was decreased in the $C c$ region soon after removal of one of the three amidases. Finally, inactivating any of the three amidases had no real impact on the intensity of the immune response to the colonization of the Pmg with L. plantarum. These data indicated that although $P G R P-S C 2$ is expressed at very 


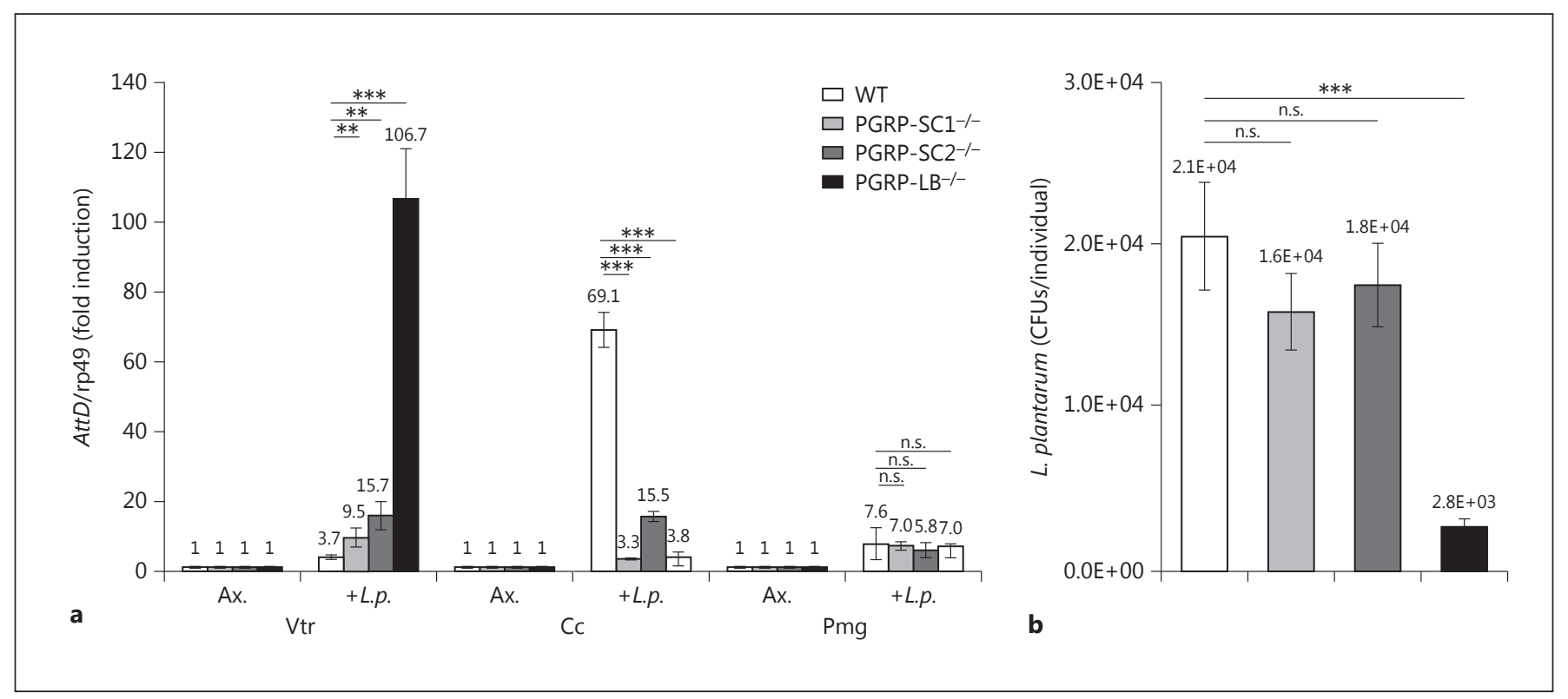

Fig. 4. $P G R P-S C 1, P G R P-S C 2$ and $P G R P-L B$ mutants have specific effects on AMP expression and bacterial load in the gut. a AttD expression in gut domains of controls (WT) and PGRP mutants recolonized by L. plantarum (L.p.). b Bacterial loads of larval guts recolonized by L. plantarum. Means \pm SD of three independent experiments. $\mathrm{p}>0.05$ (n.s.), ${ }^{* *} \mathrm{p}<0.05$ and ${ }^{* * *} \mathrm{p}<0.01$ (Student's t test). Ax. = Axenic. high levels in the gut, its absence had only limited effects on regulating IMD pathway activation after bacterial ingestion. They also highlighted the essential role of PGRP-LB in regulating IMD pathway activation in the anterior part of the gut. Since the Vtr is part of the gut that hosts gut-associated bacteria, we tested the ability of the amidase mutants to control bacterial load in the intestinal tract [29]. While mutants for either PGRP-SC1 or PGRP-SC2 had no effect on bacterial load of L. plantarum-recolonized guts, a very strong bacterial reduction was observed in PGRP-LB mutants (fig. 4b). This confirmed that PGRP-LB is the main IMD pathway regulator in the gut and that PGRP-SCs do not play a role in regulating bacterial load and intensity of immune responses in the intestinal tract.

Tissue-Specific Role of PGRP-SC2 and PGRP-LB in Dampening IMD Activation following Ecc Infection

The fact that PGRP-SC1 and PGRP-SC2 inactivation had only minor effects on local gut immune responses prompted us to test their putative implication for other host immune tissues such as the fat body. For that purpose, we turned to $E c c$, which has the ability to activate both local gut but also systemic immune responses when present in the gut, probably by releasing PGN that reach- es the hemolymph [31-33]. Diptericin (Dpt) transcription, which is a molecular readout for IMD activation in the fat body, was monitored in the three amidase mutants and compared to WT controls. Whereas feeding with Ecc had only a moderate effect on $D p t$ induction in WT and PGRP-SC1 mutants, elimination of PGRP-SC2 or PGRP$L B$ mRNAs provokes its strong up-regulation (fig. 5a). These effects could either reflect the action of PGN-degrading enzymes in the intestinal tract or in the circulating hemolymph. To distinguish between these possibilities, we monitored $D p t$ expression in flies infected with $E c c$ by pricking. Using such a protocol, the inactivation of $P G R P-S C 2$ had a much stronger effect on Dpt expression than the inactivation of PGRP-LB, which had only mild consequences (fig. 5b). Here again, the absence of PGRP$\mathrm{SC} 1$ was without any consequences. These results are well correlated with the fact that PGRP-SC2 but not PGRP-LB transcription is strongly up-regulated in the fat body of infected flies (fig. 5c, d). These results showed that while the absence of PGRP-SC1 mRNA has no effect on the level of activation of systemic immune responses, both PGRP-SC2 and PGRP-LB act as negative regulators of the IMD pathway. Interestingly, however, their effects seem to depend on the inoculation route. While PGRP-LB is needed to degrade Ecc PGN in the gut, our results suggest 


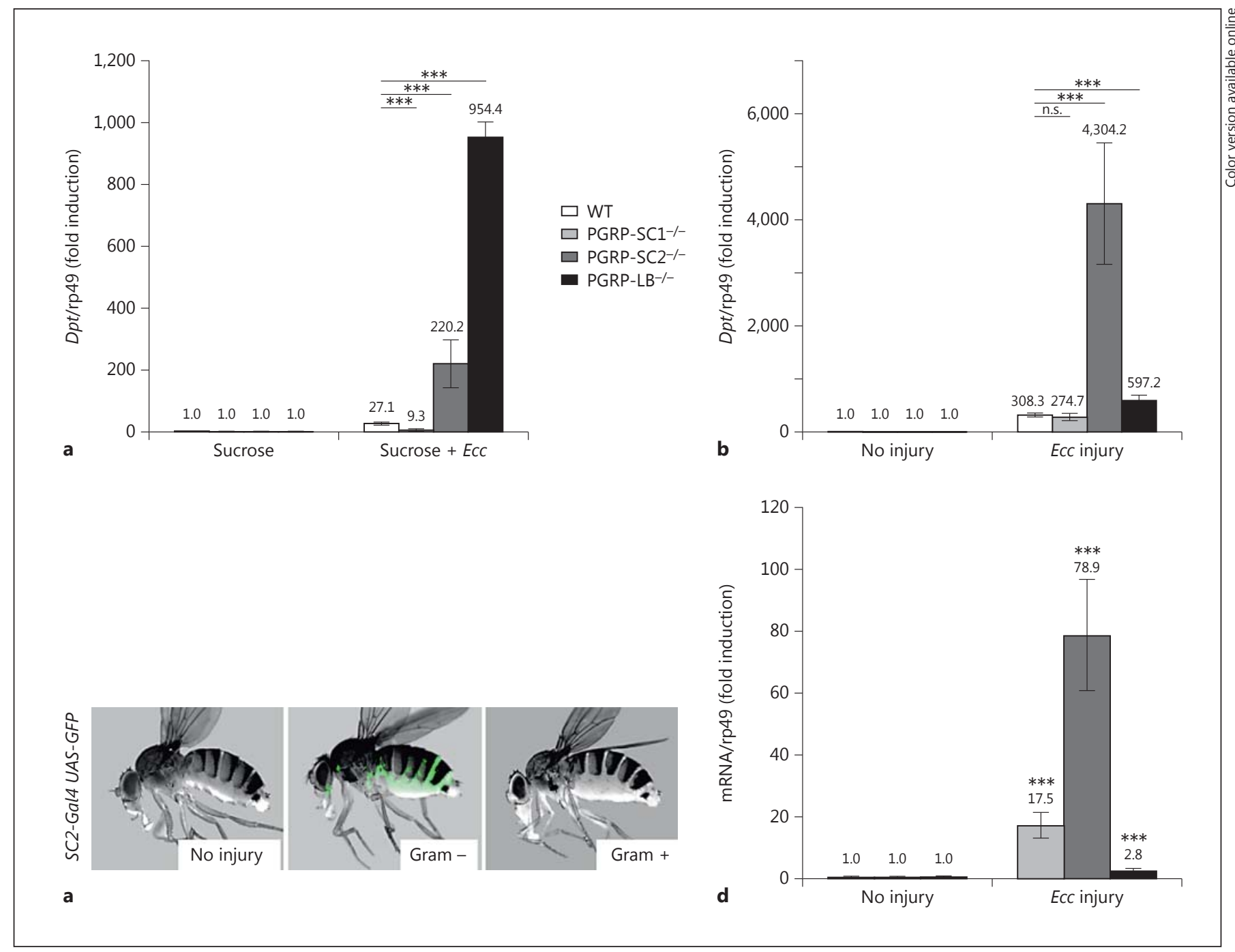

Fig. 5. The impact of $P G R P-S C 2$ and $P G R P-L B$ on IMD pathway repression after $E c c$ infection is tissue specific. Color refers to the online version only. a, b Dpt expression in WT and PGRP mutant flies $24 \mathrm{~h}$ after Ecc feeding (a) and $24 \mathrm{~h}$ after septic injury with Ecc (b). c PGRP SC2 expression in adult flies $48 \mathrm{~h}$ after septic injury.
PGRP-SC2-Gal4, UAS-nlsGFP (green) is mainly expressed in the fat body after injury with Ecc. d Induction levels of PGRP-SC1, $P G R P-S C 2$ and PGRP-LB mRNAs in the fat body of adult flies 24 $\mathrm{h}$ after Ecc injury. Means \pm SD of three independent experiments. $\mathrm{p}>0.05$ (n.s.) and ${ }^{* * *} \mathrm{p}<0.01$ (Student's t test). that PGRP-SC2 is doing so within the body cavity. To further challenge this hypothesis in vivo, we decided to compare the ability of specific amidase mutants to resist infection with the same pathogenic bacterial species inoculated via different routes.

\section{PGRP-LB and PGRP-SC2 Mutants Display Different}

Susceptibility to P. entomophila Infection Depending

on the Inoculation Route

Others and we have shown that uncontrolled IMD pathway activation is detrimental to the fly $[21,25]$. The present data indicate that while $P G R P-L B$ mutants present an exacerbated immune response when bacteria are fed to the flies, this is not the case if bacteria are inoculated in the body cavity by pricking. This contrasted with PGRP-SC2 mutants in which IMD pathway overactivation is observed with both modes of infection, although more strongly after cuticle injury. In order to test whether these differences could impact the ability of a fly to survive the detrimental effects of IMD pathway overactivation, we used $P$. entomophila spp. In contrast to $E c c, P$. entomophila is pathogenic to Drosophila but is also able 
Fig. 6. Survival rates of $P G R P-S C 1$ and PGRP-SC2 mutants after infection with bacterial pathogens. a Survival analysis of WT (Oregon-R), PGRP-SC1 mutant, PGRP-SC2 mutant, PGRP-LB ${ }^{-/-}$and $D R E D D^{-/-}$after ingestion with $P$. entomophila. PGRP-SC2 $2^{-/-}$, PGRP-LB $B^{-/-}$and $D R E D D^{-/-}$mutants are more susceptible than controls $(\mathrm{p}<0.001)$. b Survival analysis of WT (Oregon-R), PGRP-SC1 ${ }^{-/-}$, PGRP-SC2 ${ }^{-/-}$, PGRP-LB ${ }^{-/-}$and DREDD ${ }^{-/-}$ after infection with $P$. entomophila. PGRP$S C 2^{-/-}$and $D R E D D^{-/-}$mutants are more susceptible than controls ( $\mathrm{p}<0.001)$. c Survival analysis of WT (Oregon-R), PGRP-SC1 $1^{-/-}$PGRP-SC2 $2^{-/-}$and $M y d 88^{-/-}$ mutants after infection with $E$. faecalis. $\mathrm{Myd} 88^{-/-}$mutants are more susceptible than controls $(\mathrm{p}<0.001)$. Survival curves are representative of at least three independent trials. $p$ values were calculated with the log-rank and the Wilcoxon test.
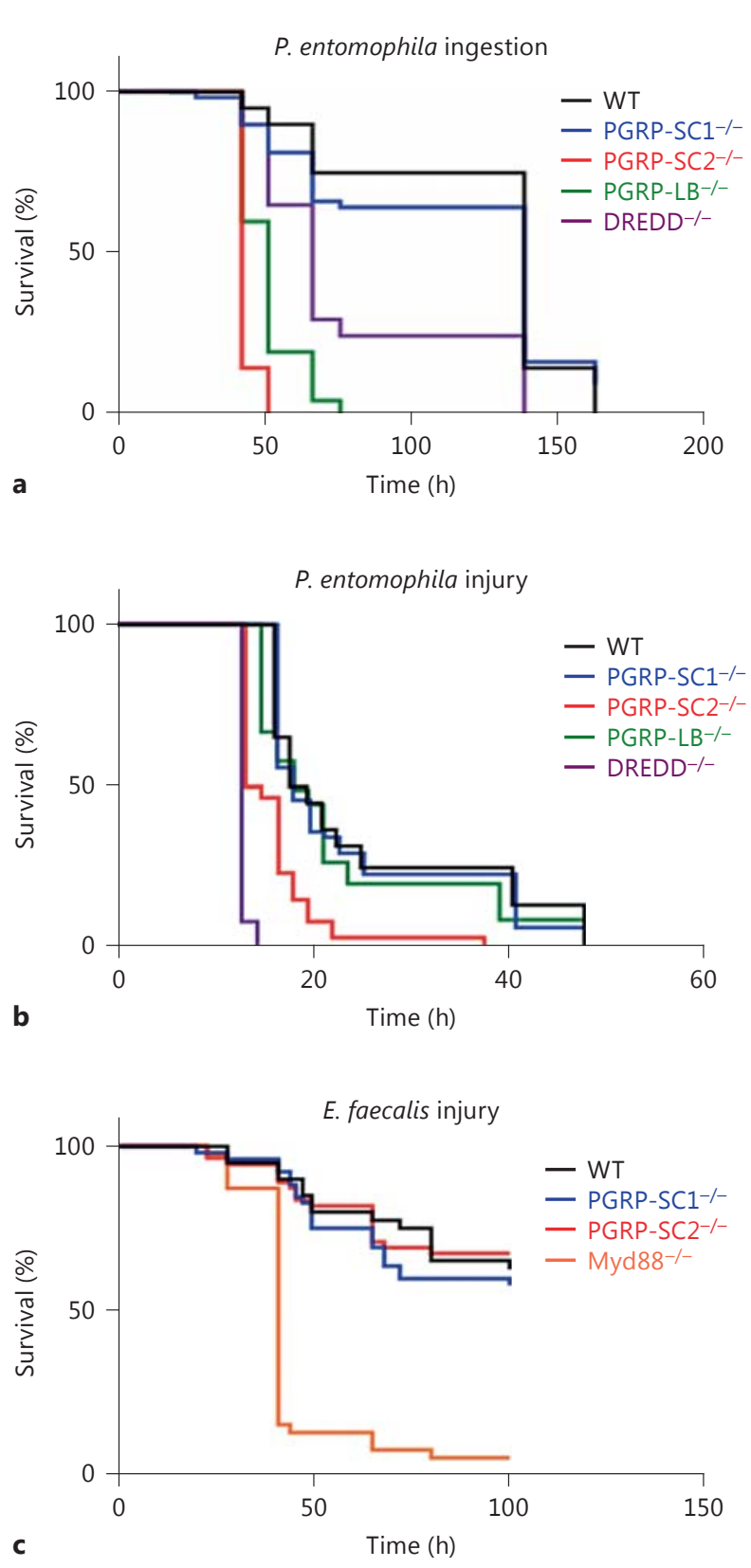

to trigger AMP production in the fat body when present in the gut [34-37]. We then compared the ability of WT and amidase-mutant flies to survive to P. entomophila infection (fig. 6a, b). Using both infection modes (oral or pricking), PGRP-SC1 mutants behave as WT controls confirming the results obtained with Ecc. Interestingly, PGRP-LB and PGRP-SC2 mutants behave differently when infected with $P$. entomophila. While both mutants showed a high mortality rate when infected orally with $P$. entomophila, only the PGRP-SC2 mutant died quicker than controls when injured with $P$. entomophila. This suggested that when PGN is present in the hemolymph, it is degraded by PGRP-SC2 to reduce IMD pathway activation. If it is in the gut, PGN is taken care of mainly by PGRP-LB. 


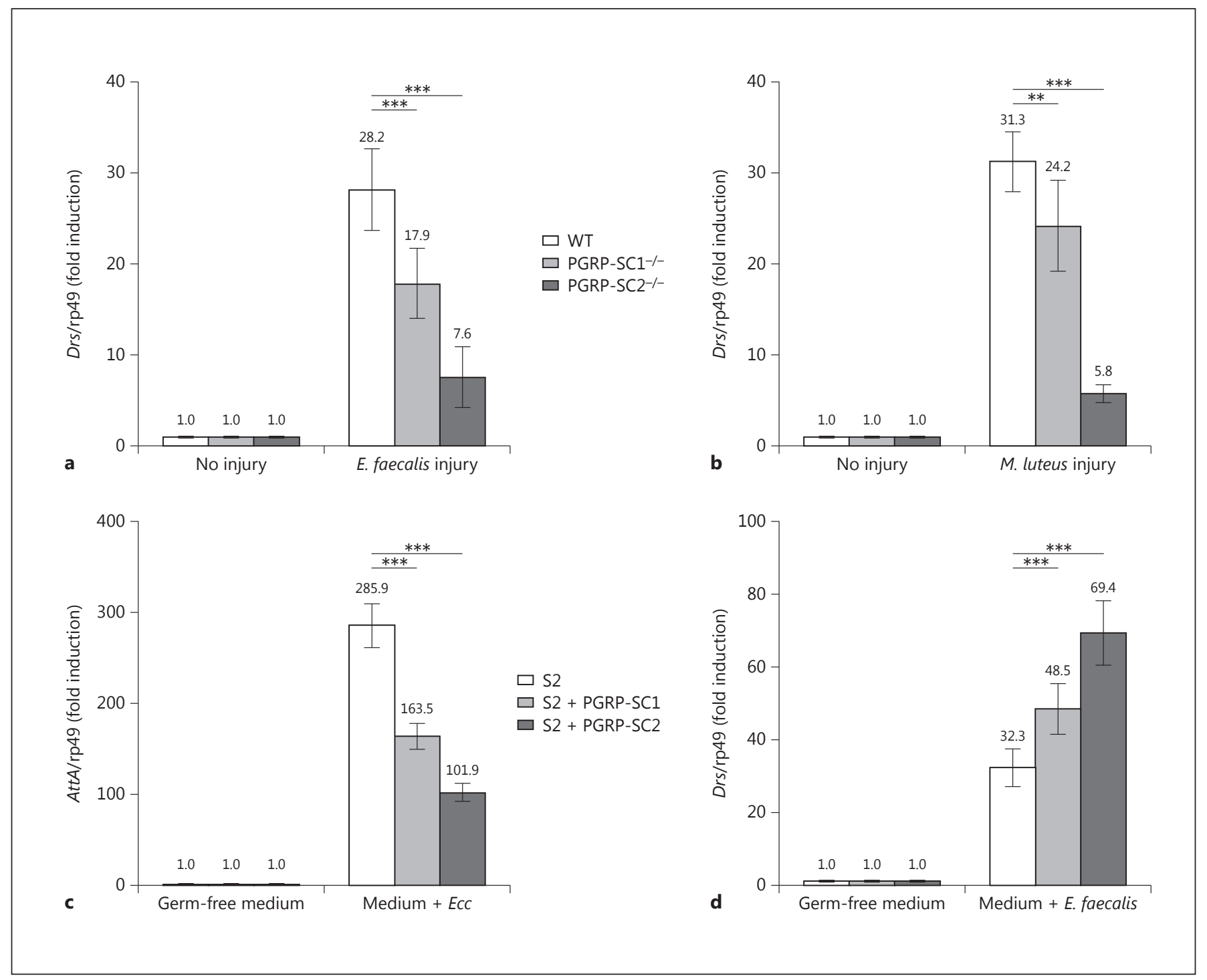

Fig. 7. Inactivation of $P G R P-S C 1$ and $P G R P-S C 2$ reduce Toll pathway activation. a, b Drs expression in controls and PGRP-SC1 and $P G R P$-SC2 mutants $24 \mathrm{~h}$ after infection with E. faecalis (a) and $M$. luteus (b). c AttA expression in S2 cells $24 \mathrm{~h}$ after addition of dead Ecc. S2 cells overexpressing PGRP-SC1 or PGRP-SC2 show de- creased AttA expression. d Drs expression in S2 cells $24 \mathrm{~h}$ after addition of dead E. faecalis. S2 cells overexpressing PGRP-SC1 or $P G R P-S C 2$ show increased Drs expression. Means \pm SD of three independent experiments. ${ }^{* *} \mathrm{p}<0.05$ and ${ }^{* * *} \mathrm{p}<0.01$ (Student's t test).
PGRP-SC1 and PGRP-SC2 Are Required for Full Toll Pathway Activation following Infection with

\section{Gram-Positive Bacteria}

In all the above tests, elimination of PGRP-SC1 had no consequence on IMD pathway activation. Taking advantage of the mutant generated, we tested whether PGRPSC1 could influence, as previously proposed [24], the activation of the Toll pathway, the second NF- $\kappa$ B signaling cascade involved in fly innate immunity. Inactivation of
PGRP-SC2, and to a lesser extent of PGRP-SC1, provoked a marked reduction in transcriptional activation of the Toll pathway target gene drosomycin (Drs) after infection with Gram-positive bacteria compared to WT controls (fig. 7a, b). This result was rather unexpected since Drosophila amidases have been proposed to cleave DAP-type PGN rather than Lys-type PGN. In addition, amidases seem here to act as facilitators of Toll pathway activation whereas they are repressors of IMD signaling. To test this 
hypothesis in a simplified system, we analyzed whether the same amidases could display antagonistic effects on Toll and IMD pathway activation in S2 cells (online suppl. fig. S2). For that purpose, we compared the ability of naïve and PGRP-SC1 - or PGRP-SC2-expressing S2 cells to activate the Toll and IMD pathways, respectively, after incubation with Gram-positive or -negative bacteria. Consistent with previous data, amidase-producing cells had a reduced ability to activate the IMD pathway compared to controls when incubated with heat-inactivated Ecc (fig. 7c). Interestingly, when the same PGRP-SC2- or PGRP-SC1-producing cells were incubated with dead $E$. faecalis, they showed an enhanced ability to activate Drs, the Toll target gene (fig. 7d). Reduction in Toll pathway activation in PGRP-SC mutants was, however, not sufficient to impair the ability of the flies to resist to E. faecalis infection (fig. 6c). These in vitro and in vivo results show that PGRP-SC1 and PGRP-SC2 exert an opposite effect on the activation of the two NF- $\mathrm{kB}$ signaling cascades. While they reduced the ability of DAP-type PGN to activate the IMD pathway, they increased the elicitor activity of Lys-type PGN on Toll pathway activation. Here, again, the effects seen with PGRP-SC2 were stronger than with PGRP-SC1.

\section{Discussion}

The results presented here demonstrate that PGRPLB, PGRP-SC1 and PGRP-SC2 have different spatiotemporal expression patterns and play specific roles in regulating Drosophila immune responses. As far as the IMD pathway is concerned, PGRP-SC1a/PGRP-SC1b elimination did not provoke any modification in immune pathway activation. This was rather unexpected, since PGRP-SC1 is the Drosophila most induced gene following bacterial colonization. In contrast, our data showed that both PGRP-SC2 and PGRP-LB are strong dampeners of the IMD pathway. In accordance with previous work, we demonstrated that PGRP-LB is the essential amidase in the gut. However, it remained unclear why the PGRP-SC2 amidase which is highly expressed in the gut has such a minor role in regulating IMD pathway activation or bacterial load in this organ. Some kind of functional redundancy could explain the lack of effect. However, previous work did not report a very strong IMD pathway up-regulation in the double $P G R P$-SC mutant [21]. In addition, whereas PGRP-LB and $P G R P-S C 2$ are both expressed in the Vtr, removing the $P G R P-L B$ gene had a clear phenotype, speaking against functional redundancy between these two PGNcleaving enzymes. In addition, using ectopic expression tools, we were able to show that while ectopic PGRP-LB expression can rescue the $P G R P-L B$-mutant phenotype, $P G R P-S C 1$ and PGRP-SC2 cannot (online suppl. fig. S3). This clearly demonstrated that in addition to being expressed in different spatiotemporal patterns, amidases are not functionally equivalent. In this respect, it is interesting to note that PGRP-LB is functionally important in the gut and PGRP-SC2 in the circulating hemolymph. Indeed, we have previously shown that the mode of bacterial detection in the gut and in the fat body are different [29]. While most enterocytes rely on the intracellular PGRP-LE for PGN detection, fat body cells detect PGN mainly via PGRP-LC. It is well possible that these two receptors are activated in vivo by different ligands. A possible model could be that PGRP-LB is preventing the production of PGRP-LE-activating ligands (such as TCT) whereas PGRP-SC2 is preventing accumulation of PGRP-LC ligands. Further experiments will be needed to test this hypothesis.

Using PGRP-SC mutants, we also showed that amidases are not only required to dampen the IMD pathway but also to facilitate Toll signaling activation. This indicated that, surprisingly, the action of amidases had opposite effects on Toll and IMD signaling activation. Since the activation of both pathways depends on PGN recognition by PGRP family members, one can postulate that while a PGRP-SC-digested DAP-type PGN will be a weaker IMD pathway activator and therefore probably a weak PGRP-LC ligand, a PGRP-SC-digested Lys-type PGN will be a good inducer of Toll signaling and therefore strongly recognized by PGRP-SA. This antagonistic effect correlates well with the fact that while the IMD cascade strongly needs to be down-regulated to prevent flies from dying of infection, this is not at all the case for the Toll pathway whose constitutive activation has no effect on the flies' viability but is probably more efficient to fight infection.

The data presented here demonstrated the complexity and interdependence of the interactions that are occurring to adapt the immune responses towards bacteria entering the body cavity of Drosophila. Analyzing immune responses in Vtr, Cc and Pmg separately, we have demonstrated that different gut domains produce different amidase cocktails and display specific responses. However, gut dissection has shown that the gut can be anatomically subdivided into more than ten subdomains [38]. This could potentially greatly increase the complexity of the regulation. In addition, one also cannot exclude
78

J Innate Immun 2016;8:67-80 DOI: $10.1159 / 000437368$
Costechareyre/Capo/Fabre/Chaduli/ Kellenberger/Roussel/Charroux/Royet 
the possibility that amidases are acting successively to degrade PGN. If such a PGN will be first cleaved by a given amidase before being a target for another PGNcleaving enzyme, the interpretation of the mutant phenotype will be even more complicated. This could be the case for Ecc PGN that could first be modified in the gut lumen by PGRP-LB before being digested in the hemolymph by PGRP-SC2. One should also keep in mind that PGRPs with amidase activity are potentially secreted proteins and could therefore act distant from the site where they are produced. They could eventually travel together with the bacteria from one gut domain to another. Finally, our data showed that mutations in a given amidase can have opposite effects on the regulation of the two main signaling immune pathways, IMD and Toll. This could potentially be explained with two biological roles of PGRP-SC, an amidase-dependent and an ami- dase-independent function. Consistently, Garver at al. [24] demonstrated that a noncatalytic cysteine-serine PGRP-SC1a transgene is able to rescue a PGRP-SC1a mutant as far as Toll pathway activation is concerned. Knowing that some immune genes are specifically activated by one cascade whereas others depend on both signaling pathways, one should interpret the immunomodulation and immune phenotypes observed in amidase mutants with caution.

\section{Acknowledgments}

We thank Fatoumata Djitte for technical assistance, and the members of Royet's laboratory for comments and discussion. This work was supported by the Equipe FRM DEQ20140329541 to Julien Royet, and by the Centre National de la Recherche Scientifique and the Agence Nationale pour la Recherche.

\section{References}

1 Royet J, Gupta D, Dziarski R: Peptidoglycan recognition proteins: modulators of the microbiome and inflammation. Nat Rev Immunol 2011;11:837-851.

-2 Royet J, Dziarski R: Peptidoglycan recognition proteins: pleiotropic sensors and effectors of antimicrobial defences. Nat Rev Microbiol 2007;5:264-277.

3 Buchon N, Silverman N, Cherry S: Immunity in Drosophila melanogaster - from microbial recognition to whole-organism physiology. Nat Rev Immunol 2014;14:796-810.

4 Kurata S: Extracellular and intracellular pathogen recognition by Drosophila PGRPLE and PGRP-LC. Int Immunol 2010;22:143148.

5 Kaneko T, Yano T, Aggarwal K, Lim JH, Ueda $\mathrm{K}$, et al: PGRP-LC and PGRP-LE have essential yet distinct functions in the Drosophila immune response to monomeric DAP-type peptidoglycan. Nat Immunol 2006;7:715723.

6 Choe KM, Lee H, Anderson KV: Drosophila peptidoglycan recognition protein LC (PGRP-LC) acts as a signal-transducing innate immune receptor. Proc Natl Acad Sci USA 2005;102:1122-1126.

7 Takehana A, Yano T, Mita S, Kotani A, Oshima Y, et al: Peptidoglycan recognition protein (PGRP)-LE and PGRP-LC act synergistically in Drosophila immunity. EMBO J 2004;23:4690-4700.

8 Werner T, Borge-Renberg K, Mellroth P, Steiner H, Hultmark D: Functional diversity of the Drosophila PGRP-LC gene cluster in the response to lipopolysaccharide and peptidoglycan. J Biol Chem 2003;278:2631926322.
-9 Michel T, Reichhart JM, Hoffmann JA, Royet J: Drosophila Toll is activated by Gram-positive bacteria through a circulating peptidoglycan recognition protein. Nature 2001;414: 756-759.

10 Leone P, Bischoff V, Kellenberger C, Hetru C, Royet J, et al: Crystal structure of Drosophila PGRP-SD suggests binding to DAP-type but not lysine-type peptidoglycan. Mol Immunol 2008;45:2521-2530.

11 Bischoff V, Vignal C, Boneca IG, Michel T, Hoffmann JA, et al: Function of the Drosophila pattern-recognition receptor PGRP-SD in the detection of Gram-positive bacteria. Nat Immunol 2004;5:1175-1180.

12 Wang L, Gilbert RJ, Atilano ML, Filipe SR, Gay NJ, et al: Peptidoglycan recognition protein-SD provides versatility of receptor formation in Drosophila immunity. Proc Natl Acad Sci USA 2008;105:11881-11886.

13 Ramet M, Manfruelli P, Pearson A, MatheyPrevot B, Ezekowitz RA: Functional genomic analysis of phagocytosis and identification of a Drosophila receptor for E. coli. Nature 2002; 416:644-648.

14 Choe KM, Werner T, Stoven S, Hultmark D, Anderson KV: Requirement for a peptidoglycan recognition protein (PGRP) in Relish activation and antibacterial immune responses in Drosophila. Science 2002;296:359-362.

15 Gottar M, Gobert V, Michel T, Belvin M, Duyk G, et al: The Drosophila immune response against Gram-negative bacteria is mediated by a peptidoglycan recognition protein. Nature 2002;416:640-644.
16 Leulier F, Parquet C, Pili-Floury S, Ryu JH, Caroff M, et al: The Drosophila immune system detects bacteria through specific peptidoglycan recognition. Nat Immunol 2003; 4 : 478-484.

17 Stenbak CR, Ryu JH, Leulier F, Pili-Floury S, Parquet C, et al: Peptidoglycan molecular requirements allowing detection by the Drosophila immune deficiency pathway. J Immunol 2004;173:7339-7348.

18 Zaidman-Remy A, Herve M, Poidevin M, Pili-Floury S, Kim MS, et al: The Drosophila amidase PGRP-LB modulates the immune response to bacterial infection. Immunity 2006; 24:463-473.

19 Zaidman-Remy A, Poidevin M, Herve M, Welchman DP, Paredes JC, et al: Drosophila immunity: analysis of PGRP-SB1 expression, enzymatic activity and function. PLoS One 2011;6:e17231.

20 Mellroth P, Steiner H: PGRP-SB1: an N-acetylmuramoyl L-alanine amidase with antibacterial activity. Biochem Biophys Res Commun 2006;350:994-999.

-21 Paredes JC, Welchman DP, Poidevin M, Lemaitre B: Negative regulation by amidase PGRPs shapes the Drosophila antibacterial response and protects the fly from innocuous

infection. Immunity 2011;35:770-779.
Mellroth P, Karlsson J, Steiner H: A scavenger function for a Drosophila peptidoglycan recognition protein. J Biol Chem 2003;278:70597064.

23 Bischoff V, Vignal C, Duvic B, Boneca IG, Hoffmann JA, et al: Downregulation of the Drosophila immune response by peptidoglycan-recognition proteins SC1 and SC2. PLoS Pathog 2006;2:e14.
Tissue-Specific Regulation of Immune Responses by Amidases 
24 Garver LS, Wu J, Wu LP: The peptidoglycan recognition protein PGRP-SCla is essential for Toll signaling and phagocytosis of Staphylococcus aureus in Drosophila. Proc Natl Acad Sci USA 2006; 103:660-665.

25 Guo L, Karpac J, Tran SL, Jasper H: PGRPSC2 promotes gut immune homeostasis to limit commensal dysbiosis and extend lifespan. Cell 2014;156:109-122.

26 Gong WJ, Golic KG: Genomic deletions of the Drosophila melanogaster Hsp70 genes. Genetics 2004;168:1467-1476.

27 Charroux B, Royet J: Elimination of plasmatocytes by targeted apoptosis reveals their role in multiple aspects of the Drosophila immune response. Proc Natl Acad Sci USA 2009; 106:9797-9802.

28 Werner T, Liu G, Kang D, Ekengren S, Steiner $\mathrm{H}$, et al: A family of peptidoglycan recognition proteins in the fruit fly Drosophila melanogaster. Proc Natl Acad Sci USA 2000;97:1377213777.
29 Bosco-Drayon V, Poidevin M, Boneca IG, Narbonne-Reveau K, Royet J, et al: Peptidoglycan sensing by the receptor PGRP-LE in the Drosophila gut induces immune responses to infectious bacteria and tolerance to microbiota. Cell Host Microbe 2012;12:153165.

30 Storelli G, Defaye A, Erkosar B, Hols P, Royet $\mathrm{J}$, et al: Lactobacillus plantarum promotes Drosophila systemic growth by modulating hormonal signals through TOR-dependent nutrient sensing. Cell Metab 2011;14:403414.

31 Quevillon-Cheruel S, Leulliot N, Muniz CA, Vincent M, Gallay J, et al: Evf, a virulence factor produced by the Drosophila pathogen Erwinia carotovora, is an S-palmitoylated protein with a new fold that binds to lipid vesicles. J Biol Chem 2009;284:3552-3562.

32 Acosta Muniz C, Jaillard D, Lemaitre B, Boccard F: Erwinia carotovora Evf antagonizes the elimination of bacteria in the gut of Drosophila larvae. Cell Microbiol 2007;9:106119.

33 Basset A, Khush RS, Braun A, Gardan L, Boccard F, et al: The phytopathogenic bacteria Erwinia carotovora infects Drosophila and activates an immune response. Proc Natl Acad Sci USA 2000;97:3376-3381.
34 Haller S, Limmer S, Ferrandon D: Assessing Pseudomonas virulence with a nonmammalian host: Drosophila melanogaster. Methods Mol Biol 2014;1149:723-740.

35 Liehl P, Blight M, Vodovar N, Boccard F, Lemaitre B: Prevalence of local immune response against oral infection in a Drosophila/ Pseudomonas infection model. PLoS Pathog 2006;2:e56.

36 Vodovar N, Vallenet D, Cruveiller S, Rouy Z, Barbe V, et al: Complete genome sequence of the entomopathogenic and metabolically versatile soil bacterium Pseudomonas entomophila. Nat Biotechnol 2006;24:673-679.

37 Vodovar N, Vinals M, Liehl P, Basset A, Degrouard J, et al: Drosophila host defense after oral infection by an entomopathogenic Pseudomonas species. Proc Natl Acad Sci USA 2005;102:11414-11419.

38 Buchon N, Osman D, David FP, Fang HY, Boquete JP, et al: Morphological and molecular characterization of adult midgut compartmentalization in Drosophila. Cell Rep 2013;3 $1725-1738$. 